\title{
ArsH protects Pseudomonas putida from oxidative damage caused by exposure to arsenic
}

The role of $\mathrm{ArsH}$ in arsenic resistance

Páez-Espino, A. David; Nikel, Pablo Ivan; Chavarría, Max; de Lorenzo, Víctor

Published in:

Environmental Microbiology

Link to article, DOI:

$10.1111 / 1462-2920.14991$

Publication date:

2020

Document Version

Peer reviewed version

Link back to DTU Orbit

Citation (APA):

Páez-Espino, A. D., Nikel, P. I., Chavarría, M., \& de Lorenzo, V. (2020). ArsH protects Pseudomonas putida from oxidative damage caused by exposure to arsenic: The role of ArsH in arsenic resistance. Environmental Microbiology, 22(6), 2230-2242. https://doi.org/10.1111/1462-2920.14991

\section{General rights}

Copyright and moral rights for the publications made accessible in the public portal are retained by the authors and/or other copyright owners and it is a condition of accessing publications that users recognise and abide by the legal requirements associated with these rights.

- Users may download and print one copy of any publication from the public portal for the purpose of private study or research.

- You may not further distribute the material or use it for any profit-making activity or commercial gain

- You may freely distribute the URL identifying the publication in the public portal 


\title{
ArsH protects Pseudomonas putida from oxidative damage \\ caused by exposure to arsenic
}

by

\section{A. David Páez-Espino1,2, Pablo I. Nikel ${ }^{3}$, Max Chavarría ${ }^{4}$ and Víctor de Lorenzo ${ }^{1 \star}$}

\begin{abstract}
1Systems Biology Program, Centro Nacional de Biotecnología (CNB-CSIC), Campus de Cantoblanco, Madrid 28049 (Spain), 2Mammoth Biosciences Inc. South San Francisco, California 94080 USA, 3The Novo Nordisk Foundation Center for Biosustainability, Technical University of Denmark, 2800, Kongens Lyngby, Denmark, 32Escuela de Química \& CIPRONA, Universidad de Costa Rica, San José, 115012060, Costa Rica.
\end{abstract}

Running title: The role of ArsH in arsenic resistance

Keywords: $\quad$ Arsenic, Pseudomonas putida, oxidative stress, ArsH

Originality-Significance Statement. The arsH gene appears systematically associated to the typical genomic clusters arsRBC that enable tolerance of many types of Gram-negative bacteria to inorganic arsenic salts. However, the specific role of ArsH in enduring either inorganic or organic As species has remained elusive_-if not contradictory_in the literature. In this work we have adopted a suite of genetic and biochemical methods to unveil thus far unknown activities of the two $\mathrm{arsH}$ paralogs found in the chromosome of the soil bacterium Pseudomonas putida. The results hereby presented demonstrate that ArsH helps to counteract the oxidative stress caused by either exposure to arsenic compounds or by unrelated insults. The arsRBCH operons thus merge direct activities against As salts (reduction and expulsion) with compensation of general physiological consequences (i.e. oxidative stress).

This article has been accepted for publication and undergone full peer review but has not been through the copyediting, typesetting, pagination and proofreading process which may lead to differences between this version and the Version of Record. Please cite this article as doi: $10.1111 / 1462-2920.14991$ 
* Correspondence to: Víctor de Lorenzo, Centro Nacional de Biotecnología (CNB-CSIC) Campus de Cantoblanco, Madrid 28049, Spain. Tel.: 34-91 58545 36. Fax: 34-91 58545 06. E-mail: vdlorenzo@cnb.csic.es 


\section{ABSTRACT}

The two As resistance arsRBC operons Pseudomonas putida KT440 are followed by a downstream gene called arsH that encodes an NADPH-dependent flavin mononucleotide reductase. In this work, we show that the arsH1 and (to a lesser extent) arsH2 genes of $P$. putida KT2440 strengthened its tolerance to both inorganic $\mathrm{As}(\mathrm{V})$ and $\mathrm{As}(\mathrm{III})$ and relieved the oxidative stress undergone by cells exposed to either oxyanion. Furthermore, overexpression of arsH1 and $\mathrm{arsH} 2$ endowed $\mathrm{P}$. putida with a high tolerance to the oxidative stress caused by diamide (a drainer of metabolic NADPH) in the absence of any arsenic. To examine whether the activity of ArsH was linked to a direct action on the arsenic compounds tested arsH1 and arsH2 genes were expressed in Escherichia coli, which has an endogenous arsRBC operon but lacks an arsH ortholog. The resulting clones both deployed a lower production of reactive oxygen species (ROS) when exposed to As salts and had a superior endurance to physiological redox insults. These results suggest that besides the claimed direct action on organoarsenicals, ArsH contributes to relieve toxicity of As species by mediating reduction of ROS produced in vivo upon exposure to the oxyanion e.g. by generating $\mathrm{FMNH}_{2}$ to fuel ROS-quenching activities.

\section{INTRODUCTION}

That virtually all types of bacteria encode in their genome one or more systems for tolerance to arsenic (As)-either in its $\mathrm{As}(\mathrm{V})$ or $\mathrm{As}(\mathrm{III})$ forms_-bears witness that dealing with this element, in particular its oxyanionic species, was an early evolutionary challenge (Silver and Phung, 2005). The core hurdle stems from the similarity of arsenate to phosphate and the high reactivity of arsenite towards sulphides (Tamaki and Frankenberger, 1992). The most straightforward way of overcoming this potential chemical dead-end, involves arsenite efflux pumps accompanied by an arsenate reductase that discriminates between $\mathrm{AsO}_{4}{ }^{3-}$ and $\mathrm{PO}_{4}{ }^{3-}$ and thus earmarks the thereby reduced (and more toxic) As species to be secreted to the external milieu (Paez-Espino et al., 2009). At the genetic level, this is reflected by the 
widespread occurrence of one or more As resistance operons (Fekih et al., 2018) with genes encoding a minimum of three activities: arsC (arsenate reductase), arsB or acr3 (efflux pump) and arsR (arseniteresponsive repressor). This basic As-resistance core is often accompanied by some additional genes that are also transcribed in response to As in the medium and thus suspect or proven to have a role in tolerance or detoxification of the oxyanions (Stolz et al., 2006; Paez-Espino et al., 2009; Yang and Rosen, 2016; Fekih et al., 2018)

The genome of the soil bacterium Pseudomonas putida KT2440 bears two separate but very similar operons for As resistance (Páez-Espino et al., 2015; Fernández et al., 2014; Fernández et al., 2016; Fig. 1A). One of them (the ars2 gene cluster) is the default ArsR system that seems to be part of the original genetic complement of this bacterium. In contrast, the ars1 cluster is part of a large insertion in the tmk gene (PP_1919, encoding a thymidylate kinase). The two systems are separately able to endow the bacterium with a high level of resistance to As, although they differ in the optimal temperature of the corresponding activities (Páez-Espino et al., 2015) —hence their consideration as ecoparalogs (Sanchez-Perez et al., 2008). Interestingly, inspection of the regions downstream of the arsRBC clusters reveals the presence in each case of an additional gene, arsH (i.e. arsH1 and arsH2; Fig. 1) that is cotranscribed with the preceding cistrons (Fernandez et al., 2014) and thus likely to have a role in As resistance as well. But what is ArsH and what does it do?

The presence of an arsH gene forming part of an extended ars operon is by no means unique of Pseudomonas, although it seems to be generally absent in Gram-positive bacteria. Apart of finding homologous sequences in the genomes of a large number of eubacteria and even yeasts (Yang and Rosen, 2016), the ArsH proteins of Shigella flexneri, Sinorhizobium meliloti and Synechocystis sp. (approximately $26 \mathrm{kDa}$ in size) have been crystalized and their tridimensional (3D) structure determined (Vorontsov et al., 2007; Ye et al., 2007; Xue et al., 2014). In other cases, the protein has been purified (to different extents) and its activities in vitro tested (Chen et al., 2015). Inspection of the protein sequences, examination of the available structures and appraisal of the enzymatic assays indicated without a doubt that ArsH is a NADPH-dependent flavin mononucleotide (FMN) reductase. However, the 
fate of the electrons thereby held by the reduced cofactor $\left(\mathrm{FMNH}_{2}\right)$ and the role of such a redox trade in As resistance remains uncertain, as the literature reports somewhat paradoxical data on the issue. First, the loss of arsH in some bacteria e.g. Serratia (Gilmour et al., 2004; Mo et al., 2011), Yersinia (Neyt et al., 1997; Eppinger et al., 2012) and Sinorhizobium, (Ye et al., 2007) results in an increased sensitivity to As. In others e.g. Thiobacillus (Butcher et al., 2000) and Synechocystis, (Hervas et al., 2012; Xue et al., 2014; Zhang et al., 2014) the lack of the enzyme seems to be alien to any As resistance $\left(A^{R}\right)$ phenotype. Second, different ArsH proteins have been shown to reduce chemical species as diverse as chromate (Xue et al., 2014), ferric iron (Mo et al., 2011; Xue et al., 2014), azo dyes (Crescente et al., 2016) and quinones (Hervas et al., 2012). The possibility that ArsH reduces molecular oxygen to $\mathrm{H}_{2} \mathrm{O}_{2}$ - which in turn can oxidize $\mathrm{AsO}_{3}{ }^{3-}$ to the less toxic $\mathrm{AsO}_{4}{ }^{3-}$ form-has been also recognized as a possible way to ease the deleterious effects of the oxyanion (Aposhian et al., 2004; Ye et al., 2007). However, it would be somewhat paradoxical that the same gene cluster that encodes reduction of arsenate to arsenite (through $\mathrm{ArsC}$ ) also enables the exactly opposite transformation (through $\mathrm{ArsH}$ ). Finally, in an unexpected twist of events, (Chen et al., 2015; Chen et al., 2018) claimed that the key ArsH activity of $P$. putida and $S$. meliloti is in fact an oxidase that provides resistance to trivalent forms of various organoarsenicals. This claim raises some questions not only regarding the enzymatic reaction involved (i.e. an NADPH-dependent FMN reductase oxidizing a given substrate). Also, arsH clearly predated the widespread use of modern organoarsenical herbicides and the poultry growth promoters tested, which could thus not act as a major selective pressure. Finally, although some ArsR variants seem to respond to methylarsenite (Chen et al., 2017), there is no evidence that such molecules are effectors of every repressor —which by default appears to respond only to inorganic arsenite (Cai et al., 1998).

On the basis of the above, we have revisited the biological function of the arsH genes of $P$. putida on the hypotheses that [i] they encode a NADPH-dependent FMN reductase, [ii] they have to be somewhat connected to $A s^{R}$ and [iii] they have to coexist with the arsenate reductase activity encoded by arsC. To this end, we have run a series of genetic, enzymatic and physiological experiments with $P$. putida variants lacking either arsH1, arsH2 or both of them. Heterologous expression of each of the genes in 
Escherichia coli and quantitative measurement of oxidative stress in a range of experimental conditions were carried out as well. The results shown below reveal that the arsH genes (in particular the one belonging to the ars1 cluster) contribute very significantly to the $A s^{R}$ phenotype of $P$. putida. Furthermore, it does so by counteracting the generic oxidative damage brought about in vivo by exposure to As species. ArsH thus appears to be part of a housekeeping mechanism geared to mitigate the physiological toxicity of the oxyanions in addition to-or instead of-a substrate-specific redox enzyme targeting organic forms of the element.

\section{RESULTS}

The two arsH genes of $P$. putida KT2440 and their products

The soil bacterium $P$. putida KT2440 is among the environmental microorganisms with the highest tolerance to As, being able to grow in the presence of up to $0.3 \mathrm{M} \mathrm{AsO}_{4}{ }^{3-}$ or $10-20 \mathrm{mM} \mathrm{AsO}_{3}{ }^{3-}$ (Fernández et al., 2014; Páez-Espino et al., 2015). This property can be traced to the presence of two coexisting ars operons (Fig. 1A) encoded in different regions of the genome and with different genealogies. Yet, they keep a comparable genomic organization and a high similarity in their sequences. As shown in Fig. 1A, both systems have also additional arsH genes (PP_1927 and $\left.P P \_2715\right)$, downstream of the respective ars $C$ sequences which have been designated arsH1 and arsH2. Inspection of the intergenic regions between ars $\mathrm{C} 1 / \mathrm{arsH} 1$ and $\operatorname{ars} \mathrm{C} 2 / \mathrm{arsH} 2$ revealed a separation between genes of 23 and 13 bp, respectively (Supplementary Fig. S1), thus signposting a degree of translational coupling indicative of co-expression in both cases.

The predicted products have also similar sizes (ArsH1, 241 amino acids and 27,7 kDa; and ArsH2, 233 amino acids and 26,6 kDa) and a high residue identity in their corresponding primary structures (Fig. 1B). When these sequences were threaded in the known tridimensional frame of the ArsH protein of $S$. meliloti (see Experimental Procedures), both ArsH1 and ArsH2 of P. putida delivered virtually identical structural predictions, with five perfectly conserved $\beta$-sheets and seven $\alpha$-helixes (Fig. 2). The only 
conspicuous difference appeared to be a small $\beta$-helix close to the $\mathrm{N}$-terminus that is kept in ArsH $S$. meliloti and $\mathrm{ArsH} 1$ P. putida but not in $\mathrm{ArsH} 2$ P. putida. Yet, this site is not anticipated to have any relevant activity. In contrast, the binding sites for NADPH and FMN that correspond to the PFAM motif PF:03358 (NADPH-dependent FMN reductase) are well kept (Supplementary Fig. S2) and thus the corresponding activities are likely to be similar or identical to that of other ArsH variants. On this background, we set out to investigate the physiological role of either ArsH variant borne by $P$. putida and their plausible involvement in As resistance in this bacterium.

ArsH1 and ArsH2 strengthen the tolerance of P. putida to arsenate and arsenite

The first obvious question regarding the biological function of the two arsH genes of $P$. putida was examining their contribution - if any-to resistance to As salts. For this, we first generated seamless deletion mutants of the arsH1 and arsH2 (either individually or in combination) in P. putida TEC1 (Table 1). This is a $\Delta$ pyrF derivative of the reference strain $P$. putida KT2442 that has been erased of the orotidine 5'-phosphate decarboxylase activity to facilitate the genome editing method described by (Galvao and de Lorenzo, 2005). Because of the pyrF deletion, the TEC1 strain is auxotrophic for uracil, but otherwise identical to the wild-type counterpart. The procedure followed for generating strains $P$. putida $\Delta a r s H 1, P$. putida $\Delta a r s H 2$ and P. putida $\Delta a r s H 1 \Delta a r s H 2$ is detailed in Experimental Procedures. Note that these deletions were designed such that they eliminated accurately the respective ORFs from the first to the last codon of the arsH ORFs, but leaving intact the corresponding upstream and downstream regions. In order to test the tolerance of the different strains to either $A s(I I I)$ or $A s(V)$, strains $\Delta a r s H 1, \Delta a r s H 2$ and $\Delta a r s H 1 \Delta a r s H 2$ were grown in LB medium added with the concentrations of the oxyanions (10-50 mM arsenate and 5-10 mM arsenite) indicated in Fig. 3 and using P. putida TEC1 as a wild-type control. The results of Fig. 3 exposed a role for both ArsH variants in tolerance to either As species, albeit to different extents. While the loss of arsH1 affected very significantly endurance to the oxyanion, the elimination of ars $\mathrm{H} 2$ had only a marginal effect, that became more evident only when compared to the extra sensitivity of the double mutant $\Delta$ arsH1 $\Delta$ arsH2. It is worth mentioning at this point that the experiments were done at $30^{\circ} \mathrm{C}$, the optimal temperature for expression 
of the ars1 operon, what may account for the differences observed between the two arsH variants (see below).

Heterologous expression of arsH1 and arsH2 increases resistance of $\mathrm{E}$. coli to arsenic

In order to ascertain whether the increment in As tolerance caused by the arsH genes in $P$. putida was essentially related to the rest of the activities encoded by the ars operons or if they had a role by themselves, we expressed the genes separately in E. coli as a surrogate host and exposed the resulting strains to As salts. To this end, each arsH gene was separately cloned in a broad-host-range expression vector, originating plasmids pVH1 $\left(\mathrm{arsH}^{+}\right)$and $\mathrm{pVH} 2\left(\mathrm{arsH}^{+}\right)$described in Table 1. E. coli JM109, used as a host for these experiments, is a derivative of the archetypal K12 strain that bears a canonical $\operatorname{arsRBC}$ lacking arsH which endows cells with a low sensitivity to inorganic arsenic species. As it was the case of $P$. putida, strains E. coli JM109 (pVH1) and E. coli JM109 (pVH2) were incubated for $48 \mathrm{~h}$ in the presence of increasing concentrations of $\mathrm{As}(\mathrm{III})$ and $\mathrm{As}(\mathrm{V})$ along with control strain E. coli JM109 (pVLT33), carrying the insert-less vector. As shown in Fig. 4, the results indicated that both ArsH1 and $\mathrm{ArsH} 2$ significantly increased tolerance to As salts. Once more, ArsH1 appeared to be more efficient than $\mathrm{ArsH} 2$ in contributing to resistance phenotype, although under certain conditions (e.g. $5 \mathrm{mM}$ arsenate or $1 \mathrm{mM}$ arsenite), the effect of $\mathrm{ArsH} 2$ was quite noticeable as well (probably the higher temperature of the experiment $-37^{\circ} \mathrm{C}-$ helped to this). The results with $P$. putida above (Fig. 3) and those with E. coli (Fig. 4) documented a separate role for the ArsH proteins in As tolerance (in particular ArsH1), whether they are produced in its native host or in another bacterium. This prompted us to examine the possible mechanisms that could account for such a phenomenon.

ArsH1 and ArsH2 contribute to the NADPH-dependent FMN reductase activity of $P$. putida

In order to verify the most obvious biological activity of the ArsH proteins suggested by the analyses of their sequences above (Fig. 1 and Fig. 2), we set out to explore their predicted oxidoreductase activity. To this end, the same four isogenic $P$. putida strains (wild-type, $\Delta$ ars $H 1, \Delta a r s H 2$ and $\Delta a r s H 1 \Delta a r s H 2$ ) 
used for the growth tests of Fig. 3 were cultured in a medium with $1 \mathrm{mM}$ arsenite to induce the transcription of the ars operons (see details in Experimental Procedures) and the biomass was lysed to generate cell-free extracts. These were used to assay the nicotinamide nucleotide-dependent flavin reductase activity. To cover as many conditions as possible, the assays were run with either FMN or FAD as substrate and NADH or NADPH as cofactor. The results are shown in Fig. 5, using in all cases the baseline activities found in the extracts of the double $\Delta$ ars $H 1 \Delta$ ars $H 2$ mutant as a reference, that allow us to establish a baseline of reductase activity corresponding to other possible enzymes or interferences. Results show that: [i] using FMN and NADPH, the reductase activity in the wild type strain is the highest ( $93 \%$ higher with respect to wt assay with FAD/NADPH, $\sim 67 \%$ higher with respect to FMN/NADH and $\sim 162 \%$ with respect FAD/NADH) and [ii] that using FMN and NADPH as cofactors, the difference of oxidoreductase activity between the wild type and the $\Delta$ arsH1 $\Delta$ ars $H 2$ mutant is also the highest $(\sim 129 \%)$ in respect to other conditions ( 90\% FAD/NADPH; $\sim 69 \%$ FMN/NADH and $\sim 0 \%$ FAD/NADH). Thus, this simple experiment with cell-free extracts of all mutant combinations indicates that the only bona fide catalytic activity that could be traced to the genes of interest was observed with FMN as a substrate and NADPH as a cofactor. Any other combination showed a much lower or absence of reductase activity. These results confirm the nicotinamide nucleotide-dependent flavin reductase activity of the purified ArsH proteins reported for $P$. putida KT2440 by Chen et al.(2015). In addition, it agrees with the dependence of cofactor (NADPH) and substrate (FMN) shown by ArsH orthologs in Shigella flexneri (Vorontsov et al., 2007), Sinorhizobium meliloti (Ye et al., 2007), Synechocystis sp. (Hervás et al., 2012) and Acidithiobacillus ferrooxidans (Hongyu et al. 2011). Fig. 5 also suggests that, while both ArsH variants contributed to the NADPH flavin oxidoreductase activity found in the cell-free extracts, the ArsH1 protein present in the extracts had a greater effect under the conditions tested: the $\mathrm{\Delta arsH} 1$ sample showed significantly less activity than the $\Delta \mathrm{ars} H 2$ counterpart. According to these data the relative hierarchy of contribution to the oxidoreductase activity detected in the tested strains using FMN as substrate and NADPH as cofactor appeared to be: TEC1 (wild-type) >> $\Delta$ arsH2 $>\Delta$ arsH1 $>\Delta$ arsH1 $\Delta a r s H 2$. This hierarchy was consistent with the more prominent role of ArsH1 in allowing growth in the presence of As salts. In view of these data, what could be the 
connection between a higher reductase activity and the enhanced tolerance to the oxyanions afforded by the ArsH proteins?

Arsenic salts triggers high intracellular levels of ROS in P. putida lacking arsH1 and arsH2

One of the known effects of exposing cells to As salts is causing oxidative stress. This is because such oxyanions can generate reactive oxygen species (ROS, e.g. hydrogen peroxide, superoxide anions, hydroxyl radicals and organic hyperoxides) that damage biomolecules, affect the respiratory chain and deplete the pool of metabolic antioxidant currency (Andres and Bertin, 2016). Bacteria have evolved a large number of strategies to deal with this type of stress and we wondered whether an enzyme that regenerates $\mathrm{FMNH}_{2}$ like ArsH could be related to counteracting oxidative damage caused by As oxyanions. The interplay of $\mathrm{FMNH}_{2}$ with ROS has not been studied in detail in bacteria, but there is evidence of it in mitochondria (Selivanov et al., 2011; Grivennikova et al., 2018) hence a role of flavin mononucleotides in dealing with redox stress appeared to be plausible. On these bases, it was of interest verifying the occurrence of ROS in As-treated $P$. putida cells bearing or not ArsH. To this end, we run the experiments shown in Fig. 6, in which cells were treated with the stressor, then added with the ROS-sensitive dye 2', 7'-dihydrochlorofluorescein diacetate $\left(\mathrm{H}_{2} \mathrm{DCF}-\mathrm{DA}\right.$, which is oxidized by ROS to DCF-DA) and the fluorescence of single cells was quantified by flow cytometry. As shown in Fig. 6, challenging wild-type $P$. putida cells with either $\mathrm{As}(\mathrm{III})$ or $\mathrm{As}(\mathrm{V})$ resulted in marginal formation of ROS, but the situation changed when mutants lacking either arsH variant were treated with the oxyanions. Consistently with the $\mathrm{As}^{\mathrm{R}}$ phenotypes of the mutants discussed above, the loss of arsH1 resulted in high levels of DCF-DA fluorescence (indicative of intracellular ROS accumulation), which were exacerbated in the double mutant $\Delta$ arsH1 $\Delta$ arsH2. The loss of arsH2 caused only a minor but still detectable increase in the ROS levels of As-treated cells. These results suggested that ArsH activity could counteract the action of oxidative damage caused by As in a fashion independent of the action of the canonical ars operon genes arsRBC. To study this possibility, we inspected the effect of the ArsH proteins on ROS stress in the absence of any arsenic, as tackled below. 
ArsH1 and ArsH2 protect P. putida from oxidative stress caused by diamide

In order to compare the endurance of $P$. putida and its ars $H$-minus variants to oxidative conditions, we adopted sensitivity to diamide (DA) as a descriptor of tolerance to redox stress. DA is a thiol-specific oxidizing agent, which reacts with thiols and protein sulfhydryls, thereby oxidizing glutathione and promoting formation of disulfide bonds (Kosower and Kosower, 1995). Typically, bacteria react to these effects by means of NADPH-dependent thioredoxins, the over-activity of which deplete NADPH (Chavarría et al., 2013). DA treatment thus triggers a factual redox stress regime, which can be either exacerbated or counteracted by the specific conditions or genetic background of the strains used. In this context, we ran the simple but informative tests shown in Fig. 7, in which a given and fixed number of cells of the $P$. putida strains TEC1, $\Delta a r s H 1, \Delta a r s H 2$, and $\Delta a r s H 1 \Delta a r s H 2$ were suspended in soft agar, lawn on the surface of an LB medium plate and then assayed for DA sensitivity in a disk test as indicated in Experimental Procedures. As shown in Fig. 7A, cells lacking separately ars $\mathrm{H} 1$ and (to an extent) $\mathrm{ars} H 2$, were clearly more sensitive to DA than the wild-type strain, the phenomenon becoming more pronounced in the double mutant $\Delta \mathrm{arsH} 1 \Delta \mathrm{arsH} 2$. Although these results were significant, note that the tests of Fig. 7A were done in the absence of any As salt, which triggers expression of the ars operons and their accompanying genes. Therefore, the effects detected had to be the result of the basal expression levels of the ars operons in the absence of bona fide induction. In order to eliminate any possible ambiguity, we repeated the same tests with $P$. putida $\Delta$ ars $H 1 \Delta$ ars $H 2$ (lacking entirely both arsH variants) transformed with plasmids $\mathrm{pVH} 1\left(\mathrm{arsH}^{+}\right)$or $\mathrm{pVH} 2\left(\mathrm{arsH} 2^{+}\right)$described in Table 1. These plasmids, which express the genes of interest through an altogether heterologous promoter were thus tested using as a control the same $\Delta$ arsH1 $\triangle$ arsH2 strain bearing the insert-less vector pVLT33. The results shown in Fig. 7B and 7C clearly exposed that mainly ArsH1 (but also ArsH2) sufficed to alleviate the redox stress caused by DA on $P$. putida in a way entirely independent of any possible effect of arsenic. The final question was whether this scenario was exclusive of $P$. putida or the redox stress counteracting role of ArsH indicated by the experiments above has a context-independent activity of its own. 
arsH1 and arsH2 enhance general tolerance of $\mathrm{E}$. coli to oxidative stress

Inspired by the results of Fig. 6 and Fig. 7, we subjected E. coli strains E. coli JM109 (pVH1, arsH1 $\left.{ }^{+}\right), E$. coli JM109 (pVH2, arsH2 $\left.{ }^{+}\right)$and arsH-less control E. coli JM109 (pVLT33, empty vector) to the test shown in Fig. 8 for the quantitative determination of ROS buildup in cells treated with DA. For this, cultures in LB medium of each strain of interest were exposed to the stressor for $6 \mathrm{~h}$ and then treated with $\mathrm{H}_{2}$ DCF-DA prior to flow cytometry analyses. The data shown in Fig. 8 clearly indicated that-as it was the case with P. putida (Fig. 6) -the ArsH proteins were not only able to check oxidative stress caused by exposure to arsenic salts, but they also counteracted the same challenge when triggered by a different environmental or metabolic condition. As evidenced in Fig. 8, ArsH1 delivered consistently better protection to stress originated by DA than ArsH2, whether the host of the genes was $E$. coli or $P$. putida. Taken together, these results pinpoint a new role of the arsH genes in resistance to As that involves an effect on downstream physiological consequences of exposure to the oxyanions rather than a direct action on the stressor molecule itself.

\section{DISCUSSION}

The role of ArsH in tolerance to As salts suggested from its systematic association to ars operons in many types of bacteria remains to this day a matter of debate. Diverse studies on the arsH genes and their products found in different microbial hosts have exposed a suite of activities of the protein that appear paradoxical at times. While some authors have demonstrated the NADPH-dependent reductase activity of ArsH on chromate (Xue et al., 2014), ferric ion (Mo et al., 2011; Xue et al., 2014), FMN (Vorontsov et al., 2007; Zhang et al., 2014), quinones (Hervas et al., 2012) and azo-dyes (Crescente et al., 2016), others have reported an specific oxidase activity of the same protein (including the variants found in P. putida) on methylarsenite and other trivalent organoarsenicals (Chen et al., 2015; Yang and Rosen, 2016). Note that the reaction FMN + NADPH $+\mathrm{H}^{+} \mathrm{FMNH}_{2}+\mathrm{NADP}^{+}$is reversible and, under specific conditions, may originate the strong oxidant FMN in vitro and in vivo. In view of this, ArsH could 
in fact be an oxidoreductase poised near equilibrium so that it can go in either direction. While its default activity could be a FMN reductase, for oxidation of organoarsenicals (Chen et al., 2015) it could behave also as a mixed-function oxidase i.e. operating with a 1:1:1 stoichiometry for pyridine nucleotide oxidation, oxygen utilization and substrate oxidation with one atom of molecular oxygen going to the substrate while the other undergoes a 2-electron reduction to water. This possibily notwithstanding, our results above show that the predominant role of the merged $\mathrm{ArsH}$ activity (i.e. $\mathrm{ArsH} 1+\mathrm{ArsH} 2$ ) of $P$. putida when cells face the oxyanion is that of a generic NADPH-dependent FMN reductase. Directly or indirectly, this activity quenches the oxidative stress caused by either exposure to As salts or brought about by another, unrelated redox-active compound such as diamide. As most of the data shown above were generated with live cells and protein extracts, we entertain that they reflect faithfully the situation in the natural context. But, how can ArsH behave as a reductase under some circumstances and as an oxidase in others? Could this dual activity also manifest itself in living cells as well? One recent report claimed an oxidative activity of ArsH in the periplasm, which seems to increase the tolerance to arsenite of various environmental isolates including some Pseudomonas species (Chang et al., 2018). Halomonas has also arsH as part of an efficient $\mathrm{As}(\mathrm{III})$ and $\mathrm{As}(\mathrm{V})$ resistance system which lacks arsenate reductase altogether (Wu et al., 2018). Chances that arsH gene has evolved specifically to deal with organoarsenicals are thus arguable, although it can indeed give rise to an increased tolerance to these compounds in vivo and originate oxidized forms in vitro. Flavoproteins are typically involved in electron transactions in the respiratory chain (Muras et al., 2019) the membrane in which they reside mediates a split in metabolism into a more oxidative environment (the periplasm) and into a reductive location (the cytoplasm). One scenario worth to explore is one in which ArsH contributes both to keep a strong oxidative environment in the periplasm and to help maintaining redox homeostasis and ROS endurance in the cytoplasm when cells are challenged with various As species. In this respect, it is intriguing ArsH seems to appear preferentially in diderm, periplasm-bearing Gram-negative bacteria and not in Gram-positive species. Yet, the molecular mechanisms underlying such a potential division of biochemical roles in the same cell cannot be directly drawn from the largely genetic data above. Finally, we cannot rule out additional effects of accompanying genes other than arsH located upstream and 
downstream of the core arSRBC clusters and which in many cases are conserved in a variety of species (Páez-Espino et al., 2009). Their specific functions in arsenic tolerance do deserve future studies.

\section{EXPERIMENTAL PROCEDURES}

\section{Bacterial strains, culture conditions, and general procedures}

All bacterial strains and plasmids used in the present work are listed in Table 1. P. putida and E. coli strains were grown at $30^{\circ} \mathrm{C}$ and $37^{\circ} \mathrm{C}$, respectively, in lysogeny broth (LB) rich medium (Sambrook, 1989) in an orbital shaker at $170 \mathrm{rpm}$. P. putida strains were also grown in M9 minimal medium (Sambrook, 1989) with sodium citrate (2 g/liter) as the carbon source and supplemented with $\mathrm{MgSO}_{4}(2$ $\mathrm{mM}$ ). When M9 was utilized for $E$. coli cultivations, glucose was added $0.2 \%$ (w/v) as a carbon source, as well as thiamine $[0.02 \%(\mathrm{w} / \mathrm{v})]$, casaminoacids $[0.1 \%(\mathrm{w} / \mathrm{v})]$ and $\mathrm{CaCl}_{2}(10 \mu \mathrm{M})$. Experiments in Petri dishes were conducted with the corresponding culture media (rich or minimal) added with $1.5 \%(w / v)$ agar. Antibiotics were used at the following concentrations: ampicillin (Ap), $150 \mu \mathrm{g} / \mathrm{ml}$; kanamycin (Km) $50 \mu \mathrm{g} / \mathrm{ml}$; and chloramphenicol (Cm), $30 \mu \mathrm{g} / \mathrm{ml}$. In order to grow P. putida $\Delta p y r F$ strains, uracil (SigmaAldrich Co.) was added to the plates at $20 \mu \mathrm{g} / \mathrm{ml}$. 5'-Fluoroorotic acid (FOA, Zymo Research) was used at $250 \mu \mathrm{g} / \mathrm{ml}$ to counterselect for the pyrF activity (orotidine-5'-phosphate decarboxylase, see details below). Resistance to As species was grossly tested by plating serial dilutions of cultures of each strain onto agar plates containing filtered sodium arsenite $\left(\mathrm{NaH}_{2} \mathrm{AsO}_{3}\right)$ or sodium arsenate $\left(\mathrm{NaH}_{2} \mathrm{AsO}_{4}\right)$, as necessary for the experiment at stake. For a more precise determination of minimal inhibitory concentrations (MICs) and growth rates across all the experimental conditions, strains were cultured in 96-well microtiter plates and incubated at their optimal temperature for $24 \mathrm{~h}$ with orbital shaking, and the optical density measured at $600 \mathrm{~nm}\left(\mathrm{OD}_{600}\right)$ values were assessed using an Ultrospec-3000 pro spectrophotometer (Pharmacia Biotech). All oligonucleotides employed for the assembly of the various constructs and strains were synthesized by Sigma-Aldrich Co. and detailed below.

\section{Mutants and genetic constructs}


Plasmids and cloning procedures were handled following the standard methods described in (Sambrook, 1989). All P. putida mutants were obtained using TEC1 (KT2442 $\triangle$ pyrF::xylE: Table 1) as a the parental strain. Strain TEC1 is auxotrophic for uracil and resistant to FOA. The method used for gene deletion was an adaptation of the Saccharomyces cerevisiae URA3 selection system and is fully detailed in (Galvao and de Lorenzo, 2005). For single deletion mutants in arsH1 and arsH2, the whole arsH genes were separately deleted as follows. Upstream and downstream regions of each arsH gene were amplified by PCR producing the so called Up and Down fragments. The pairs of primers used in each case were ForwH1Up (5'-CCA CCA GCG GCC GCT CGG CAT CGG TTT CAG CGA GTA CG3')/RevH1Up (5'-TGA TCC AGG GCC TAC AGC GCG-3') and ForwH2Up (5'-TCA ACT GCG GCC GCA TAT TTT CGC TGG GCA TGT ATC TGG TGG-3')/RevH2Up (5'-GTT TTC TTC CTG TTC AAA GCG AGC CGA T-3') to generate Up regions of asrH1 and arsH2 genes respectively. ForwH1Down (5'-CGC GCT GTA GGC CCT GGA TCA GAT AAT GAG CAT GTC GCA TCC TTA CTC ATT G-3')/RevH1Down (5'-GTC CCG AGC TCC GTT GCG CTG ATG ACG ACA CGA G-3') and ForwH2Down (5'-ATC GGC TCG CTT TGA ACA GGA AGA AAA CAG CAA AGG TGA TTC AAG ACA GTG GAA ACG3')/RevH2Down (5'-GTC CCG AGC TCT GGC CAA TGT CAT CCG CAG GCG-3') were utilized to acquire the corresponding Down regions. In both cases, the Rev-Up oligo was complementary to the Forw-Down counterpart. This way, we obtained the Up and Down fragments in a first PCR reaction that were then amplified with the most external oligos (Forw-Up and Rev—Down) in a second PCR. The resulting Up-Down fragment for each arsH gene was therefore flanked by Notl and Sacl restriction sites. These fragments were inserted into the cloning vector pTEC $\left(\mathrm{Km}^{\mathrm{R}}, \mathrm{R} 6 \mathrm{~K}\right.$ conditional origin, $\left.p y r \mathrm{~F}^{+}\right)$ to generate plasmids pTUDH1 and pTUDH2. They were then transformed into $E$. coli CC118 $\lambda$ pir strain via tri-parental mating using E. coli HB101 (pRK600) strain as a helper and TEC1 as receptor. TEC1 cointegrates were selected in M9-citrate and $\mathrm{Km}$ and resolved by growing a pool of them in LB medium supplemented with uracil (12 $\mathrm{h}$ in liquid culture). 100- $\mu$ l aliquots of such cultures were plated in M9citrate with uracil and FOA to counterselect all the co-integrates. The resultant colonies were checked by PCR, and a 50-50\% mixture of wild-type TEC1 strains and mutants in the corresponding arsH gene was found. The final phenotype of the mutants was uracil-, FOAR, $\mathrm{Km}^{\mathrm{s}}$ (Supplementary Fig. S3). To 
create the double $\Delta$ arsH1 $\Delta$ arsH2 deletion mutant, the same protocol was followed using the plasmid pTUDH2 in the mating with $P$. putida $\triangle a r s H 1$ as the receptor strain. For construction of the plasmids used in the complementation studies, each of the two arsH genes was separately amplified with primers that added EcoRI and HindllI sites upstream and downstream respectively, of the cognate arsH genes. To this end, we employed the oligos set FWDH1 (5'-CGA CGG AAT TCG CGC GCT GTA GGC CCT GGA TC-3') and RVSH1 (5'-TCA GCG AAG CTT GCG ACA TGC TCA TTA TCT CAA ATA GAC CG-3)' for the arsH1 gene, as well as FWDH2 (5'-CTT CGG AAT TCC TGG ATC GCA TCG GCT CGC TTT G3') and RVSH2 (5'-TCA GAG AAG CTT CGT TTC CAC TGT CTT GAA TCA CCT TTG C-3') for the arsH2 counterpart. The thereby generated products were cloned as EcoRI-HindllI fragments (including their own RBS) in the broad-host-range vector pVLT33 (de Lorenzo et al., 1993; Table 1) yielding plasmids pVH1 (arsH1+) and pVH2 (arsH2+), respectively (further details in Supplementary Fig. S4).

\section{ArsH enzymatic assays}

All samples used in the enzymatic assays were prepared from $50-\mathrm{ml}$ cultures of $P$. putida strains TEC1, $\Delta$ arsH1, $\Delta$ arsH2 and $\Delta$ arsH1 $\Delta$ arsH2 in LB medium supplemented with uracil and $1 \mathrm{mM} \mathrm{As}(\mathrm{III})$. Cultures grown until stationary phase were centrifuged at $4,000 \mathrm{rpm}$ and $4^{\circ} \mathrm{C}$ during $10 \mathrm{~min}$ and the pellets resuspended in a buffered phosphate buffer solution (PBS). The whole biomass of the $50 \mathrm{ml}-$ cultures at $\mathrm{OD}_{600} \sim 0.8$ were re-suspended in $2 \mathrm{ml}$ of PBS buffer. Then, cells were lysed (by sonication at low temperature) and centrifuged at 14,000 rpm during $30 \mathrm{~min}$. The supernatant was used for the enzymatic assays and for determining protein content with the Bradford (1976) method. NAD(P)H:flavin oxidoreductase assays were conducted as described in (Vorontsov et al., 2007) in 96-wells plates, monitoring the decrease in the absorbance at $340 \mathrm{~nm}\left(\mathrm{NAD}(\mathrm{P}) \mathrm{H}, \varepsilon=6.22 \mathrm{mM}^{-1} \mathrm{~cm}^{-1}\right)$ at $25^{\circ} \mathrm{C}$ during $10 \mathrm{~min}$. The enzymatic activity was indicated in Units per milligram of total protein (U/mg protein). The enzymatic assays were performed in $200 \mu$ l (final volume) with a reaction mix composed of $150 \mu \mathrm{M}$ $\mathrm{NADH}$ or NADPH, $100 \mu \mathrm{M}$ FMN or FAD, $25 \mathrm{mM}$ Tris- $\mathrm{HCl}(\mathrm{pH}=7.5)$ and $75 \mu \mathrm{g}$ of total protein (either obtained from the wild-type and arsH mutants strains). 
Quantification of reactive oxygen species in vivo and oxidative stress plate tests

Intracellular accumulation of ROS in P. putida TEC1 and its derivatives was followed with the ROSsensitive green fluorescent dye $\mathrm{H}_{2}$ DCF-DA (Sigma-Aldrich Co.) as explained in (Akkaya et al., 2018). The same method was adopted for inspecting ROS accumulation in $\mathrm{E}$. coli producing arsH-encoded proteins heterologously. In brief, cultures of the wild-type P. putida TEC1 strain and the arsH1 and arsH2 single and double mutants were incubated in LB medium in the presence of $\mathrm{As}(\mathrm{III})$ or (V) salts at the concentrations and times indicated. Whether P. putida or E. coli, after the treatment cells were collected by centrifugation, washed once with PBS and resuspended in in the same buffer to an $\mathrm{OD}_{600}$ 0.4. The suspension was added with $\mathrm{H}_{2}$ DCF-DA to $20 \mu \mathrm{M}$ and incubated in the dark for 30 min at room temperature. Cells were then washed twice with PBS, resuspended again in the same buffer PBS and analyzed by flow cytometry. The $\mathrm{H}_{2}$ DCF-DA fluorescence emission at $525 \mathrm{~nm}$ was detected using a 530/30-nm band pass filter array. Semi-quantitative assays for detecting sensitivity to oxidative stress with diamide were run as follows. LB plates added with $\mathrm{Km}$ for ensuring plasmid retention were spread with a homogeneous suspension of the strains under study in melted soft $0.75 \%$ (w/v) LB agar. Filter discs $(5 \mathrm{~mm} \varnothing)$ were placed onto the homogeneous bacterial lawn and soaked with $10 \mu \mathrm{l}$ of $100 \mathrm{mM}$ diamide. The plates were incubated at $30^{\circ} \mathrm{C}$ during $24 \mathrm{~h}$ and photographed. The diameter of the inhibition haloes were then considered a proxy of the sensitivity of the strains at stake to the oxidative stress caused by the drain of NADH brought about by diamide.

\section{ACKNOWLEDGEMENTS}

Authors are indebted to Barry Rosen and Luis Lopez-Maury for critical reading of the manuscript and many useful comments. This work was funded by the MADONNA (H2020-FET-OPEN-RIA-2017-1766975), BioRoboost (H2020-NMBP-BIO-CSA-2018), SYNBIO4FLAV (H2020-NMBP/0500) and MIXUP (H2020-Grant 870294) Contracts of the European Union and the S2017/BMD-3691 InGEMICS-CM Project of the Comunidad Autónoma de Madrid (European Structural and Investment Funds). The financial support from The Novo Nordisk Foundation (grant NNF10CC1016517, and LiFe, 
NNF180C0034818) and the Danish Council for Independent Research (SWEET, DFF-Research Project 8021-00039B) to P.I.N. is gratefully acknowledged. Authors declare no conflict of interest.

\section{Supplementary Materials}

Supplementary Figure S1. Intergenic regions ars $\mathrm{C}$-arsH in the arsRBCH genomic loci of $P$. putida KT2440.

Supplementary Figure S2. Structural prediction of the NADPH-dependent FMN reductase protein domain of the ArsH1 protein of Pseudomonas putida KT2440.

Supplementary Figure S3. Generation and verification of single arsH knock out mutants.

Supplementary Figure S4. Assembly and verification of $\mathrm{arsH}^{+}$plasmids.

\section{REFERENCES}

Akkaya, Ö., Pérez-Pantoja, D.R., Calles, B., Nikel, P.I., and de Lorenzo, V. (2018) The metabolic redox regime of Pseudomonas putida tunes its evolvability toward novel xenobiotic substrates. mBio 9: e01512-01518.

Andres, J., and Bertin, P.N. (2016) The microbial genomics of arsenic. FEMS Microbiol Rev 40: 299322.

Aposhian, H.V., Zakharyan, R.A., Avram, M.D., Sampayo-Reyes, A., and Wollenberg, M.L. (2004) A review of the enzymology of arsenic metabolism and a new potential role of hydrogen peroxide in the detoxication of the trivalent arsenic species. Toxicol Appl Pharmacol 198: 327-335.

Bradford, M.M. (1976) A rapid and sensitive method for the quantitation of microgram quantities of protein utilizing the principle of protein-dye binding. Anal Biochem 72: 248-254. 
Butcher, B.G., Deane, S.M., and Rawlings, D.E. (2000) The chromosomal arsenic resistance genes of Thiobacillus ferrooxidans have an unusual arrangement and confer increased arsenic and antimony resistance to Escherichia coli. Appl Environ Microbiol 66: 1826-1833.

Cai, J., Salmon, K., and DuBow, M.S. (1998) A chromosomal ars operon homologue of Pseudomonas aeruginosa confers increased resistance to arsenic and antimony in Escherichia coli. Microbiology 144: 2705-2713.

Chang, J.-S., Yoon, I.-H., and Kim, K.-W. (2018) Arsenic biotransformation potential of microbial arsH responses in the biogeochemical cycling of arsenic-contaminated groundwater. Chemosphere 191: 729-737.

Chavarría, M., Nikel, P.I., Pérez-Pantoja, D., and de Lorenzo, V. (2013) The Entner-Doudoroff pathway empowers Pseudomonas putida KT2440 with a high tolerance to oxidative stress. Environ Microbiol 15: 1772-1785.

Chen, J., Bhattacharjee, H., and Rosen, B.P. (2015) ArsH is an organoarsenical oxidase that confers resistance to trivalent forms of the herbicide monosodium methylarsenate and the poultry growth promoter roxarsone. Mol Microbiol 96: 1042-1052.

Chen J., Nadar V.S., Rosen B.P. (2017) A novel MAs(III)-selective ArsR transcriptional repressor. Mol Microbiol 106: 469-478

Chen J., Yoshinaga M., Rosen B.P. (2018). The antibiotic action of methylarsenite is an emergent property of microbial communities. Mol Microbiol 111: 487-494

Crescente, V., Holland, S.M., Kashyap, S., Polycarpou, E., Sim, E., and Ryan, A. (2016) Identification of novel members of the bacterial azoreductase family in Pseudomonas aeruginosa. Biochem $\mathrm{J}$ 473: 549-558.

de Lorenzo, V., Eltis, L., Kessler, B., and Timmis, K.N. (1993) Analysis of Pseudomonas gene products using lac/9/Ptrp-lac plasmids and transposons that confer conditional phenotypes. Gene 123: $17-24$. 
Eppinger, M., Radnedge, L., Andersen, G., Vietri, N., Severson, G., Mou, S. et al. (2012) Novel plasmids and resistance phenotypes in Yersinia pestis: unique plasmid inventory of strain Java 9 mediates high levels of arsenic resistance. PloS One 7: e32911.

Fekih, I.B., Zhang, C., Li, Y.P., Zhao, Y., Alwathnani, H.A., Saquib, Q. et al. (2018) Distribution of arsenic resistance genes in prokaryotes. Front Microbiol 9: 2473.

Fernández, M., Morel, B., Ramos, J.L., and Krell, T. (2016) Paralogous regulators ArsR1 and ArsR2 of Pseudomonas putida KT2440 as a basis for arsenic biosensor development. Appl Environ Microbiol 82: 4133-4144.

Fernández, M., Udaondo, Z., Niqui, J.L., Duque, E., and Ramos, J.L. (2014) Synergic role of the two ars operons in arsenic tolerance in Pseudomonas putida KT2440. Environ Microbiol Rep 6: 483489.

Galvao, T.C., and de Lorenzo, V. (2005) Adaptation of the yeast URA3 selection system to gramnegative bacteria and generation of a \{delta\}betCDE Pseudomonas putida strain. Appl Environ Microbiol 71: 883-892.

Gilmour, M.W., Thomson, N.R., Sanders, M., Parkhill, J., and Taylor, D.E. (2004) The complete nucleotide sequence of the resistance plasmid R478: defining the backbone components of incompatibility group $\mathrm{H}$ conjugative plasmids through comparative genomics. Plasmid $\mathbf{5 2}$ : 182-202.

Grivennikova, V.G., Kareyeva, A.V., and Vinogradov, A.D. (2018) Oxygen-dependence of mitochondrial ROS production as detected by Amplex Red assay. Redox Biol 17: 192-199.

Herrero, M., de Lorenzo, V., and Timmis, K.N. (1990) Transposon vectors containing non-antibiotic resistance selection markers for cloning and stable chromosomal insertion of foreign genes in Gram-negative bacteria. J Bacteriol 172: 6557-6567.

Hervas, M., Lopez-Maury, L., Leon, P., Sanchez-Riego, A.M., Florencio, F.J., and Navarro, J.A. (2012) ArsH from the cyanobacterium Synechocystis sp. PCC 6803 is an efficient NADPHdependent quinone reductase. Biochemistry 51: 1178-1187. 
Hongyu, M., Chen, Q., Du, J., Tang, L., Qin, F., Miao, B., Wu, X. and Zeng, J. (2011) Ferric Reductase Activity of the ArsH Protein from Acidithiobacillus ferrooxidans. J. Microbiol. Biotechnol 21: 464-469.

Kessler, B., de Lorenzo, V., and Timmis, K.N. (1992) A general system to integrate lacZ fusions into the chromosomes of gram-negative eubacteria: regulation of the Pm promoter of the TOL plasmid studied with all controlling elements in monocopy. Mol Gen Genet 233: 293-301.

Kosower, N.S., and Kosower, E.M. (1995) Diamide: An oxidant probe for thiols. Meth Enzymol 251: 123133.

Mo, H., Chen, Q., Du, J., Tang, L., Qin, F., Miao, B. et al. (2011) Ferric reductase activity of the ArsH protein from Acidithiobacillus ferrooxidans. J Microbiol Biotechnol 21: 464-469.

Muras, V., Toulouse, C., Fritz, G., and Steuber, J. (2019) Respiratory Membrane Protein Complexes Convert Chemical Energy. In Bacterial Cell Walls and Membranes: Springer, pp. 301-335.

Nelson, K.E., Weinel, C., Paulsen, I.T., Dodson, R.J., Hilbert, H., Martins dos Santos, V.A. et al. (2002) Complete genome sequence and comparative analysis of the metabolically versatile Pseudomonas putida KT2440. Environ Microbiol 4: 799-808.

Nikel, P.I., Romero-Campero, F.J., Zeidman, J.A., Goñi-Moreno, Á., de Lorenzo, V. (2015) The glyceroldependent metabolic persistence of Pseudomonas putida KT2440 reflects the regulatory logic of the GIpR repressor. mBio 6: pii: e00340-15

Neyt, C., Iriarte, M., Thi, V.H., and Cornelis, G.R. (1997) Virulence and arsenic resistance in Yersiniae. J Bacteriol 179: 612-619.

Paez-Espino, D., Tamames, J., de Lorenzo, V., and Canovas, D. (2009) Microbial responses to environmental arsenic. Biometals 22: 117-130.

Páez-Espino, A.D., Durante-Rodríguez, G., and de Lorenzo, V. (2015) Functional coexistence of twin arsenic resistance systems in Pseudomonas putida KT2440. Environ Microbiol 17: 229-238.

Sambrook, J., T. Maniatis, and T. Fritsch (1989) Molecular Cloning: a Laboratory manual. N.Y.

Sanchez-Perez, G., Mira, A., Nyiro, G., Pasic, L., and Rodriguez-Valera, F. (2008) Adapting to environmental changes using specialized paralogs. Trends Genet 24: 154-158. 
Selivanov, V.A., Votyakova, T.V., Pivtoraiko, V.N., Zeak, J., Sukhomlin, T., Trucco, M. et al. (2011) Reactive oxygen species production by forward and reverse electron fluxes in the mitochondrial respiratory chain. PLoS Comp Biol 7: e1001115.

Silver, S., and Phung, L.T. (2005) Genes and enzymes involved in bacterial oxidation and reduction of inorganic arsenic. Appl Environ Microbiol 71: 599-608.

Stolz, J.F., Basu, P., Santini, J.M., and Oremland, R.S. (2006) Arsenic and selenium in microbial metabolism. Annu Rev Microbiol 60: 107-130.

Tamaki, S., and Frankenberger, W.T., Jr. (1992) Environmental biochemistry of arsenic. Rev Environ Contam Toxicol 124: 79-110.

Vorontsov, I.I., Minasov, G., Brunzelle, J.S., Shuvalova, L., Kiryukhina, O., Collart, F.R., and Anderson, W.F. (2007) Crystal structure of an apo form of Shigella flexneri ArsH protein with an NADPH-dependent FMN reductase activity. Prot Sci 16: 2483-2490.

Wu, S., Wang, L., Gan, R., Tong, T., Bian, H., Li, Z. et al. (2018) Signature arsenic detoxification pathways in Halomonas sp. strain GFAJ-1. mBio 9: e00515-00518.

Xue, X.M., Yan, Y., Xu, H.J., Wang, N., Zhang, X., and Ye, J. (2014) ArsH from Synechocystis sp. PCC 6803 reduces chromate and ferric iron. FEMS Microbiol Lett 356: 105-112.

Yang, H.-C., and Rosen, B.P. (2016) New mechanisms of bacterial arsenic resistance. Biomed J 39: 513.

Yanisch-Perron, C., Vieira, J., and Messing, J. (1985) Improved M13 phage cloning vectors and host strains: nucleotide sequences of the M13mp18 and pUC19 vectors. Gene 33: 103-119.

Ye, J., Yang, H.-C., Rosen, B.P., and Bhattacharjee, H. (2007) Crystal structure of the flavoprotein ArsH from Sinorhizobium meliloti. FEBS Letters 581: 3996-4000.

Zhang, X., Xue, X.M., Yan, Y., and Ye, J. (2014) Purification, crystallization and preliminary X-ray diffraction analysis of ArsH from Synechocystis sp. strain PCC 6803. Acta Crystallogr $F$ Struct Biol Commun 70: 497-500. 
Table 1. Bacterial strains and plasmids used in this work.

\begin{tabular}{|c|c|c|}
\hline Strains & Relevant characteristics & Reference \\
\hline P. putida KT2440 & P. putida mt-2 derivative cured of pWW0 & (Nelson et al., 2002) \\
\hline P. putida TEC1 & RifR $^{2}, \Delta p y r F:: x y \mid E$ derivative of $P$. putida KT2442 & $\begin{array}{r}\text { (Galvao and de Lorenzo, } \\
\text { 2005) }\end{array}$ \\
\hline P. putida $\Delta$ arsH1 & $\begin{array}{l}\text { RifR, TEC1 deleted of the arsH1 gene by using } \\
\text { plasmid pTUDH1 }\end{array}$ & This work \\
\hline P. putida $\Delta$ arsH2 & $\begin{array}{l}\text { RifR, TEC1 deleted of the arsH2 gene by using } \\
\text { plasmid pTUDH2 }\end{array}$ & This work \\
\hline P. putida $\Delta$ ars $H 1 \Delta$ ars $H 2$ & $\begin{array}{l}\text { RifR, TEC1 deleted of arsH1 and arsH2 genes by } \\
\text { sequential use of plasmids pTUDH1 and pTUDH2 }\end{array}$ & This work \\
\hline E. coli CC118 $\lambda$ pir & E. coli CC118 lysogenized with $\lambda$ pir phage & (Herrero et al., 1990) \\
\hline E. coli HB101 & 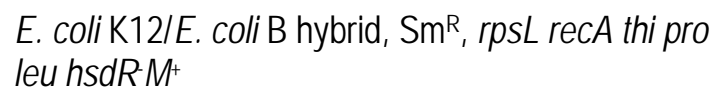 & (Sambrook, 1989) \\
\hline E. coli JM109 & 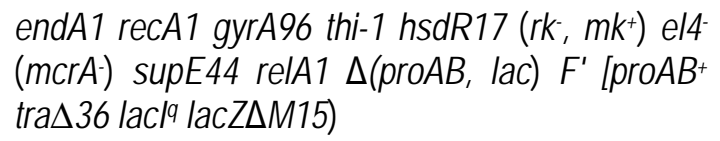 & $\begin{array}{r}\text { (Yanisch-Perron et al., } \\
\text { 1985) }\end{array}$ \\
\hline
\end{tabular}

\begin{tabular}{|c|c|c|}
\hline Plasmids & & \\
\hline pVLT33 & $\begin{array}{l}\mathrm{Km}^{\mathrm{R}}, \mathrm{RSF} 1010 \text { oriV lac/a/Ptac broad-host-range } \\
\text { expression vector with pUC18 multiple cloning } \\
\text { site }\end{array}$ & (de Lorenzo et al., 1993) \\
\hline pTEC & $\begin{array}{l}\mathrm{Km}^{\mathrm{R}}, \mathrm{FOA}^{\mathrm{S}}, \text { pyrF }^{+}\left(\mathrm{Ura}^{+}\right), \mathrm{R} 6 \mathrm{KoriV} \text {, origin of } \\
\text { transfer RK2 oriT, cloning vector for chromosomal } \\
\text { integration/deletion by homologous recombination } \\
\text { in P. putida TEC1 }\end{array}$ & $\begin{array}{r}\text { (Galvao and de Lorenzo, } \\
\text { 2005) }\end{array}$ \\
\hline pRK600 & $\begin{array}{l}\mathrm{Cm}^{\mathrm{R}} \text {, ColE1ori, RK2mob, RK2tra, helper of } \\
\text { conjugal transfer }\end{array}$ & (Kessler et al., 1992) \\
\hline pTUDH1 & $\begin{array}{l}\mathrm{Km}^{\mathrm{R}} \text {, FOA }, \mathrm{pyrF}^{+}\left(\mathrm{Ura}^{+}\right) \text {; pTEC vector inserted } \\
\text { with a } 2.17-\mathrm{kb}^{2} \mathrm{Notl} / \mathrm{Sacl} \mathrm{DNA} \text { segment composed } \\
\text { of } 1.27-\mathrm{kb} \text { upstream and } 0.90-\mathrm{kb} \text { downstream } \\
\text { regions of the arsH1 gene. These regions were }\end{array}$ & This work \\
\hline
\end{tabular}


amplified by PCR using FWDH1Up/RVSH1Up and FWDH1Down/RVSH1Down as primer sets, respectively. Delivery vector for $\Delta \mathrm{ars} H 1$ deletion.

pTUDH2

$\mathrm{Km}^{\mathrm{R}}, \mathrm{FOA}^{\mathrm{S}}, \mathrm{pyrF}^{+}\left(\mathrm{Ura}^{+}\right)$; pTEC vector inserted This work with a 1.71-kb Notl/Sacl DNA segment composed of $0.94-\mathrm{kb}$ upstream and $0.77-\mathrm{kb}$ downstream regions of the arsH1 gene. These regions were amplified by PCR using FWDH2Up/RVSH2Up and FWDH2Down/RVSH2Down as primer sets, respectively. Delivery vector for $\triangle \mathrm{ars} H 2$ deletion.

pVH1

$\mathrm{Km}^{\mathrm{R}}, \mathrm{pVLT33}$ vector inserted with the gene arsH1

This work from strain TEC1 cloned by PCR as an EcoRI/HindIII 0.72-kb long DNA fragment using primers FWDH1 and RVSH1.

pVH2

$\mathrm{Km}^{\mathrm{R}}$, pVLT33 vector inserted with the gene arsH2 This work from strain TEC1 cloned by PCR as an EcoRI/HindIII 0.71-kb long DNA fragment using primers FWDH2 and RVSH2.

Abbreviations: $\quad$ Rif, rifampicin; Cm, chloramphenicol; Km, kanamycin; Sm, streptomycin; FOA, 5'-fluoroorotic acid; URA, uracil. 


\section{CAPTIONS TO FIGURES}

Figure 1. The ArsH proteins of $P$. putida KT2440. (A) Alignment of amino acid sequences. Percentage of sequence identity of amino acids throughout the corresponding genomic regions is shown. The products of the genes arsH1 (PP_1927) and arsH2 (PP_2715) of P. putida KT2440 are annotated in the genome as proteins involved in arsenic resistance. (B) Comparison of ArsH protein variants of $P$. putida KT2440. Both proteins are very similar, having a size of 241 amino acids and a molecular weight of 27,717 Da for ArsH1, and 233 amino acids and 26,616 Da for ArsH2. Using double alignments between protein sequences with the application of BLAST (bl2seq), the high amino acid identity between both sequences could be observed. Their conserved NADPH-reductase domain dependent on FMN (PF: 03358) is highlighted in green.

Figure 2. Representation of the three-dimensional structure of the monomers ArsH of $P$. putida KT2440. Predicted 3D structures of the (A) ArsH1 and (B) ArsH2 proteins of $P$. putida KT2440. In all cases, $\alpha$ helixes, $\beta$-sheets and loops are indicated in red, yellow and green, respectively. $(C)$ Alignment of the structures of $\mathrm{ArsH} 1$ (in yellow) and $\mathrm{ArsH} 2$ (in red) of $P$. putida KT2440 with ArsH of S. meliloti (in blue). Using the protein modeling of the PyMOL program and the comparison of structures with UCSF Chimera (https://www.cgl.ucsf.edu/chimera), we found that the five $\beta$-sheets and the seven $\alpha$-helices are perfectly conserved with respect to reference protein of $S$. meliloti. The detail of the helix residue not present in $\mathrm{ArsH} 2$ near the $\mathrm{N}$-terminal end is highlighted with a purple circle.

Figure 3. Growth of strains $P$. putida TEC1, P. putida $\triangle \operatorname{ars} H 1, P$. putida $\triangle a r s H 2$ and $P$. putida $\triangle a r s H 1$ $\Delta \mathrm{arsH} 2$ at different concentrations of $\mathrm{As}(\mathrm{V})$ and $\mathrm{As}(\mathrm{III})$. Graphs $(\mathrm{A})$ and $(\mathrm{C})$ show the growth of the indicated strains against different $\mathrm{As}(\mathrm{V})$ concentrations at $8 \mathrm{~h}$ and $24 \mathrm{~h}$, respectively, while (B) and (D) indicate the same with $\mathrm{As}(\mathrm{III})$. The growth experiments were performed at $30^{\circ} \mathrm{C}$ in 96 -well plates, using LB medium supplemented with uracil and added with $\mathrm{As}(\mathrm{V})$ or $\mathrm{As}(\mathrm{III})$ as indicated. The graphs show the results of three biological replicates and two technical duplicates and error bars represent the standard deviation of the average values. $\mathrm{OD}_{600}$, optical density measured at $600 \mathrm{~nm}$. 
Figure 4. Arsenic resistance of $E$. coli JM109 expressing separately each of the two arsH genes of $P$. putida KT2440. The experiments were by incubating cells for $48 \mathrm{~h}$ at $37^{\circ} \mathrm{C}$ in 96 -well plates using medium LB with $\mathrm{Km}$ added and the arsenic species indicated. The graphs show the results of three biological replicates and two technical duplicates per condition, and error bars represent the standard deviation of the average values. $\mathrm{OD}_{600}$, optical density measured at $600 \mathrm{~nm}$.

Figure 5. Oxidoreductase activity assay in $P$. putida TEC1 and its $\triangle a r s H$ variants. The graphs show the activity of the wild-type strain TEC1 (A), $\triangle \mathrm{arsH} 1$ (B) and $\triangle \mathrm{arsH} 2(\mathrm{C})$, using the background activity of the double mutant under different substrates (FMN or FAD) and cofactors (NADPH or NADH) as a reference. The specific activity ( $\mathrm{U} / \mathrm{mg}$ total protein) of each reaction is shown, and grey bars correspond to the double mutant $\Delta \mathrm{arsH} 1 \mathrm{AarsH} 2$. The graphs indicate the results of three biological replicates and two technical duplicates per condition, and error bars represent the standard deviation of the average values.

Figure 6. Quantitative analysis of ROS accumulation in P. putida TEC1 and its arsH mutants upon exposure to arsenic. (A) Representative results of 2',7'-dichlorofluorescein diacetate (DCF-DA) fluorescence at the single cell level in the strains under study as assessed by flow cytometry. Cultures of the wild-type P. putida TEC1 strain (wt, wild-type) and the $\triangle \mathrm{arsH} 1$ and $\triangle \mathrm{arsH} 2$ single and double mutants were incubated in LB medium in the presence of $10 \mathrm{mM} \mathrm{As}(\mathrm{III})$ for $24 \mathrm{~h}$ at $30^{\circ} \mathrm{C}$. The grey rectangle in this figure identifies the range of values of green fluorescence considered to be background (i.e. basal fluorescence). (B) Quantification of ROS formation. Cultures of the wild-type $P$. putida TEC1 strain and the arsH1 and arsH2 single and double mutants were incubated in the presence of As(III) at $10 \mathrm{mM}$ or $\mathrm{As}(\mathrm{V})$ at $50 \mathrm{mM}$ for $24 \mathrm{~h}$. The percentage of DCF-DA+ cells was analyzed for each condition in triplicates from at least four independent cultures. The values shown were corrected by the background fluorescence observed in non-stained controls. Box plots represent the median value and the 1st and 3rd quartiles of the percentage of DCF-DA+ cells. Significant differences when compared to the wildtype strain $(P<0.05)$ were assessed with the Mann-Whitney $U$ test, as noted with asterisk symbols $\left(^{\star}\right)$. The color code for the $P$. putida strains is the same for the two panels. 
Figure 7. Evaluation of redox stress sensitivity in arsH mutants derived from P. putida TEC1. (A) Relative diamide sensitivity of the arsH single and double mutants. The relative sensitivity was scored as the area of inhibition, and this parameter was normalized to the area of inhibition of $P$. putida TEC1 (indicated by a broken gray line). Each bar represents the mean value of relative inhibition zone $\pm S D$ from at least two independent experiments carried in triplicates. (B) Complementation assay in which $P$. putida $\triangle \mathrm{arsH} 1 \Delta \mathrm{arsH} 2$ was supplemented with either arsH gene in trans and submitted to redox stress by exposure to diamide. The double arsH mutant transformed with the empty pVLT33 plasmid was used as a control (Ctrl.). Results from duplicated assays are shown. (C) Quantification of the relative diamide sensitivity in the complementation assays. The relative sensitivity was calculated as indicated above, and this parameter was compared to that of $P$. putida $\Delta$ arsH1 $\Delta$ arsH2 complemented with the empty pVLT33 plasmid (indicated by a broken gray line). Each bar represents the mean value of relative inhibition zone \pm standard deviation from at least two independent experiments carried in triplicates. Note that expression or either arsH1 or arsH2 restores (to an extent) the tolerance to diamide of the double knock-out strain.

Figure 8. Quantitative analysis of ROS accumulation in E. coli JM109 carrying arsH variants upon exposure to diamide. Quantification of ROS formation in cultures of E. coli JM109 strain carrying either arsH1 or ars $\mathrm{H} 2$ in the presence of diamide at $5 \mathrm{mM}$ for $6 \mathrm{~h}$. The percentage of 2',7'-dichlorofluorescein diacetate (DCF-DA)+ cells was determined by counting the fraction of cells (triplicates from at least five independent cultures) displaying fluorescence levels above the threshold set as indicated in Fig. 6 (see Nikel et al., 2015 for details of the method). Box plots represent the median value and the 1st and 3rd quartiles of the percentage of DCF-DA+ cells, and the black asterisk symbols (**) identify significant differences at $P<0.01$ tested with the Mann-Whitney $U$ test as compared to the untreated control strain. The blue asterisk symbol (*) identifies a significant difference at $P<0.05$ tested with the Mann-Whitney $U$ test as compared to the control strain in the presence of diamide. 


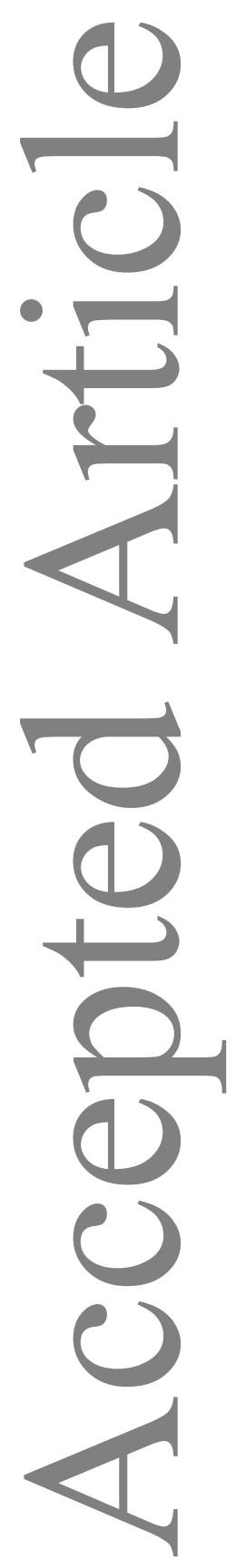

亡

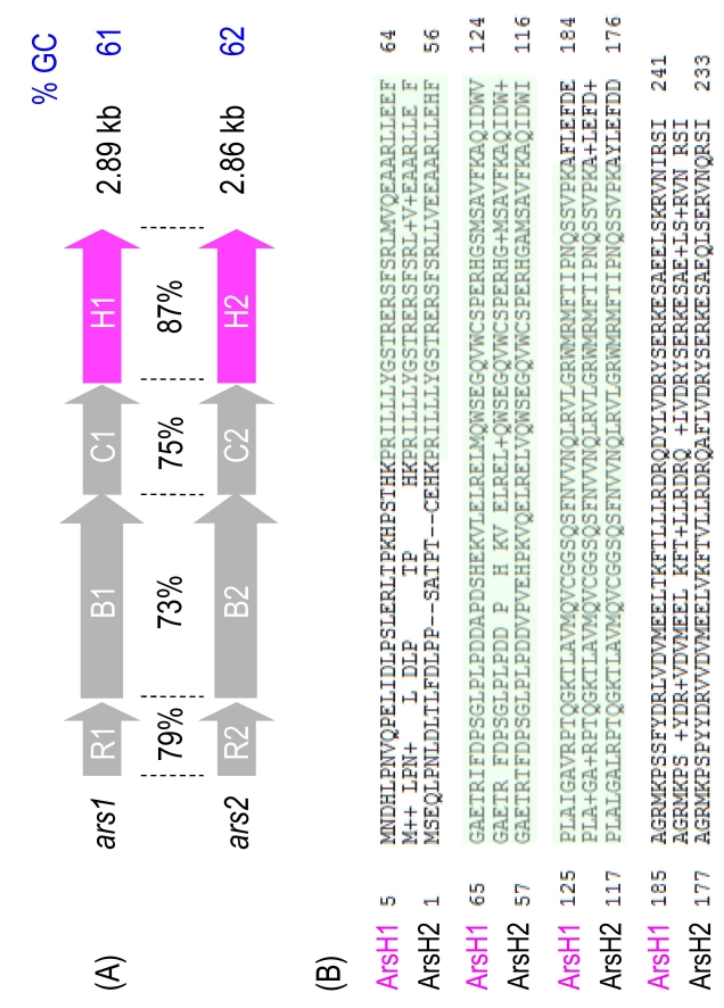

Figure 1

This article is protected by copyright. All rights reserved. 


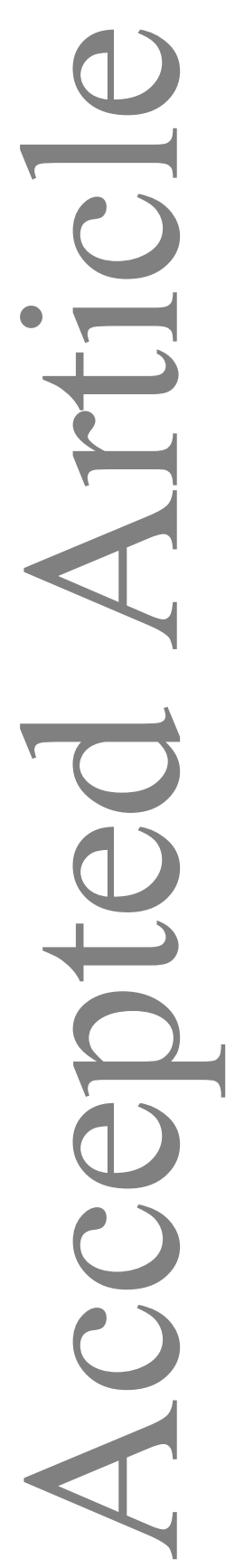

亡
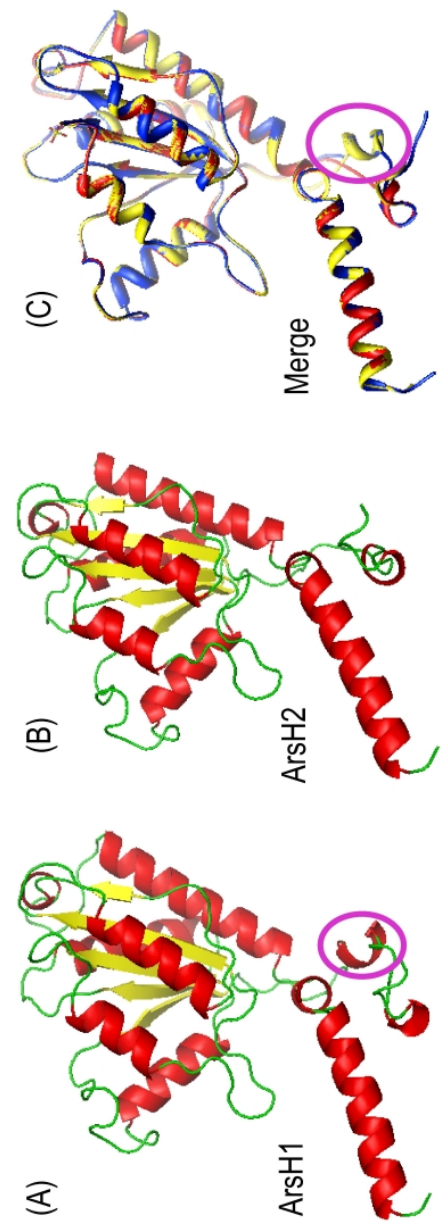

Figure 2 
以

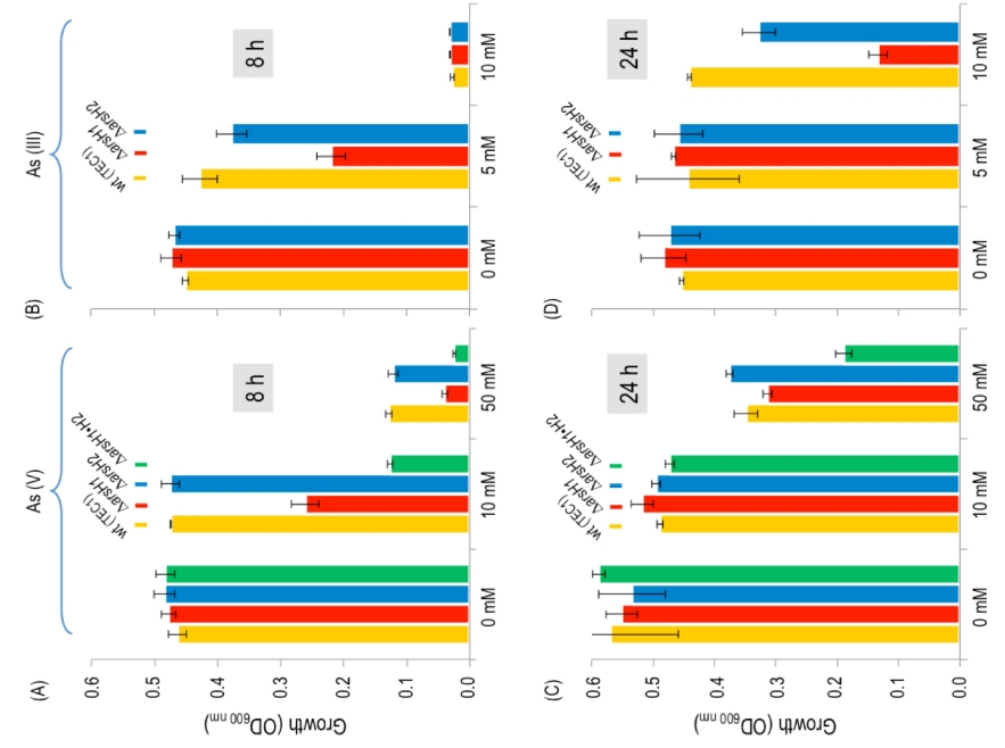

Figure 3

This article is protected by copyright. All rights reserved. 


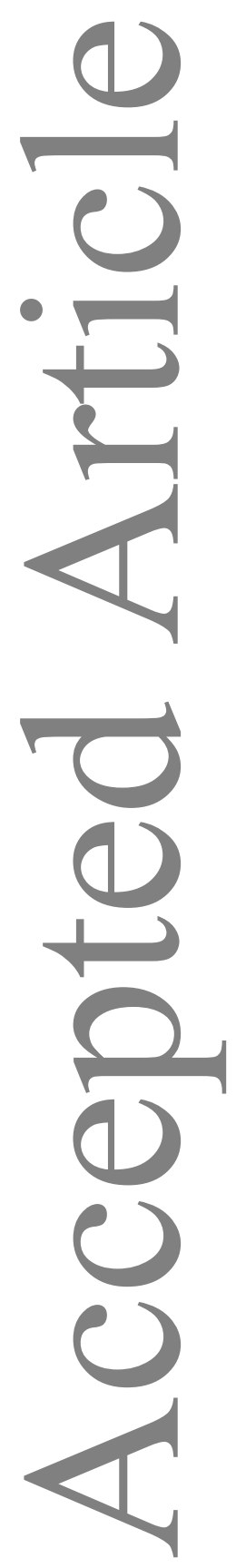

サ
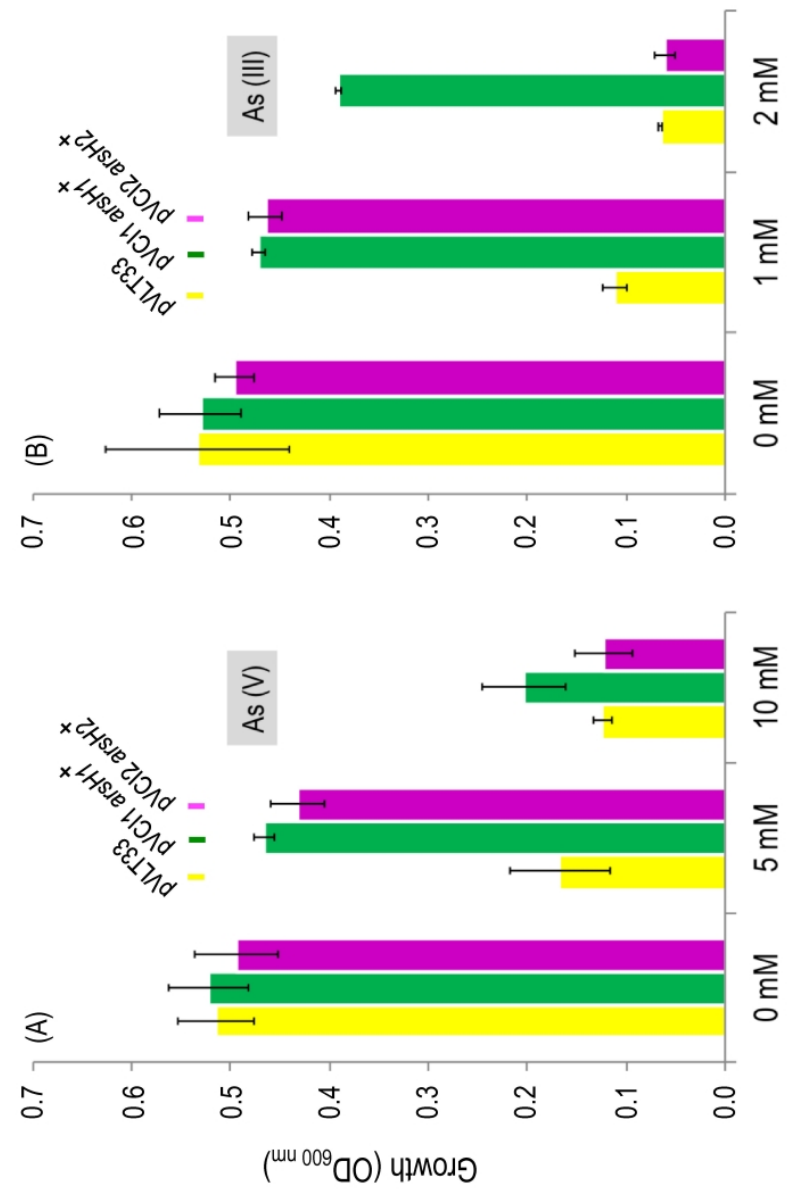

Figure 4

This article is protected by copyright. All rights reserved. 


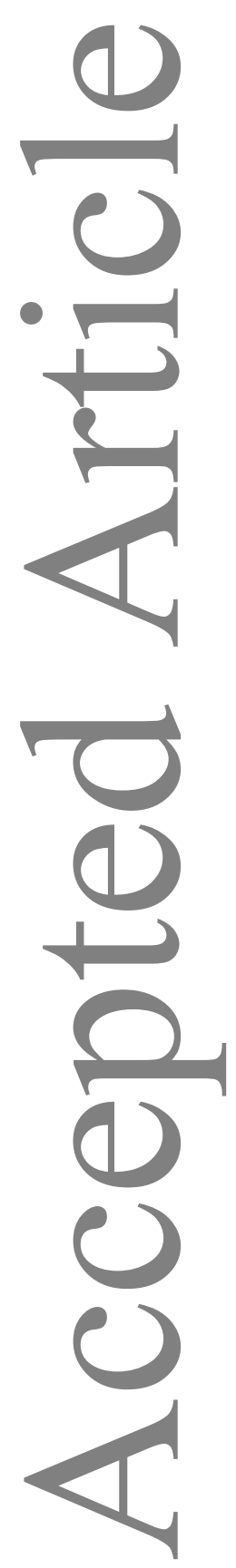

는
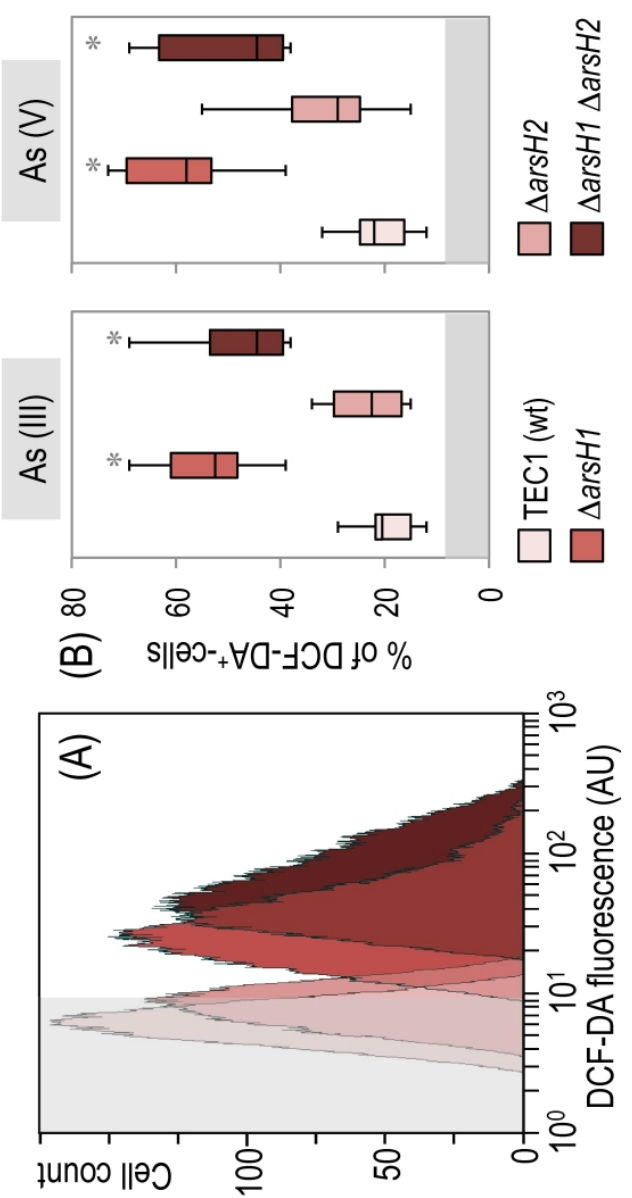

Figure 6 

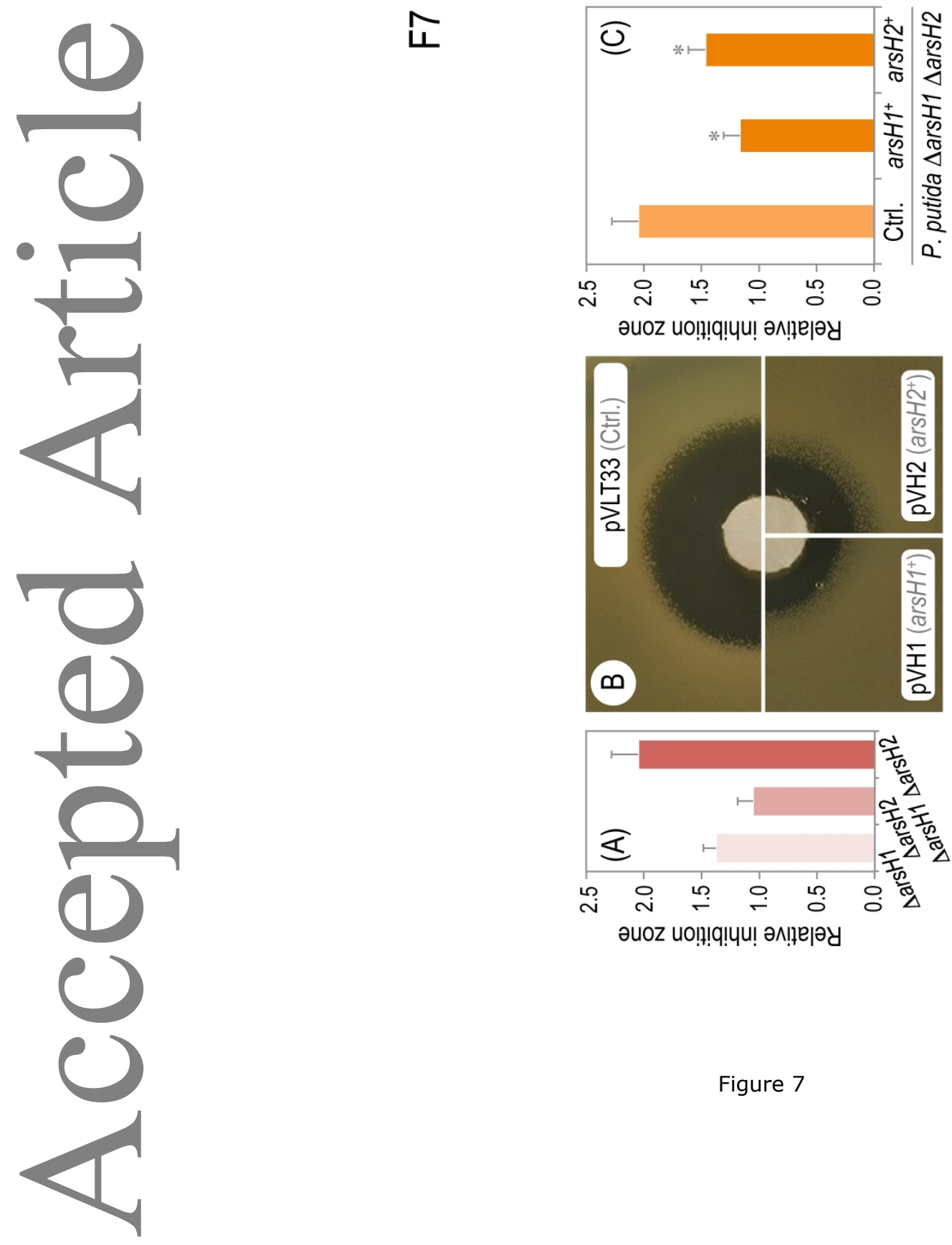

Figure 7 
